\title{
Transformation of a Thin Gold Film to Nanoparticles after Nanosecond - Laser Irradiation
}

\author{
Karolis RATAUTAS ${ }^{* 1}$, Mindaugas GEDVILAS ${ }^{* 1}$, Bogdan VOISIAT ${ }^{* 1}$, Gediminas \\ RAČIUKAITIS ${ }^{* 1}$, Alfonsas GRIGONIS ${ }^{* 2}$ \\ ${ }^{* 1}$ Center for Physical Sciences and Technology, Savanoriu Ave. 231, LT-02300 Vilnius, Lithuania \\ E-mail: karolis.ratautas@ftmc.lt \\ *2 Department of Physics, Kaunas University of Technology, Studentu 50, LT-3031 Kaunas, \\ Lithuania
}

\begin{abstract}
Dynamics of nanoparticle formation were observed after nanosecond laser irradiation of thin gold films. Gold films of different thickness $(3,4,5,6,10,15,20,25 \mathrm{~nm})$ were evaporated on the silicon (110) substrate and irradiated with the pulsed nanosecond laser using different pulse energies and the number of pulses in a burst. A single Gaussian beam as well as an interference of a few laser beams were used for irradiation. Morphological changes appeared in the films only when the pulse energy was high enough to initiate the phase transition. The threshold energy density for phase transitions in the films was estimated from the thermal model of the laser beam and sample interaction. With the pulse energy just above the threshold, it was possible to observe evolution of nanoparticle formation from a plane metal film by changing the number of pulses applied, as duration of the pulse burst represented the time the liquid phase existed. The final size of nanoparticles was a function of the film thickness and was found to be weakly dependent on the pulse energy and the number of pulses. The nanoparticle size distribution width was much narrower after irradiation with periodical intensity distribution produced by 3 interfering beams compared with the single beam treatment. Moreover, the nanoparticles tended to be periodically distributed on the substrate.
\end{abstract}

DOI: $10.2961 / \mathrm{jlmn} .2012 .03 .0022$

Keywords: metal film, laser irradiation, laser beam interference, dewetting, nanoparticles, size distribution

\section{Introduction}

Many new areas of gold nanoparticles application have appeared recently. Gold nanoparticles on a silicon substrate are used in catalytic structural growth processes [1], or in production of carbon nanotubes or silicon nanowires.

Nanoparticles as a catalyst can be used for anisotropic etching [2]. Therefore, they are an important structural part in a variety of sensors [3] (gene-sensor, immuno-sensor, electro-catalytic, chemo-sensor). Moreover, nanoparticles are used for signal amplification [ 4 ] in Raman spectroscopy and in other technological areas.

The applications require controllable distribution of nanoparticles on substrates. Formation of regular arrays of nanoparticles was investigated in [5] by thermal annealing of the $\mathrm{Au}$ film on pre-patterned substrates. Direct laser irradiation of thin metal films on flat substrates leads to nanoparticle formation as well [6], although such process requires plenty of time. Henley et al. [7] investigated nanoisland formation from thin metal film after nanosecond laser annealing, and as result the diameter of nanoislands strongly depended on the film thickness. Therefore, it is important to understand the physical processes which lead to nanoparticle formation. From the technological point of view, it is important to estimate the final size of nanoparticles and their area density theoretically from laser process and film parameters.
There are two physical phenomena, which can cause nanoparticle formation in thin films on a substrate under pulsed laser heating: a) heterogeneous nucleation from liquid or gaseous phase with location on defects and contaminations and b) rupture of a film due to hydrodynamic spinodal instability in a thin film - spinodal dewetting [6]. In the heterogeneous nucleation process, a film rupture is initiated by interaction of defects (or other heterogeneities) with film atoms. Each of these processes differently reacts to changes in physical conditions: temperature, temperature gradient, time spent at high temperature.

Spinodal dewetting occurs when a liquid film becomes unstable due to the film - substrate and film - film intermolecular interaction [6]. Then intermolecular forces reach the value of the surface tension force, perturbations in a thin liquid film (initiated by spinodal hydrodynamic instability) growth in time and the film becomes unstable [8]. The thin-film hydrodynamic equation, which is based on the lubrication approximation of the nonlinear NavierStokes equations, was applied to evaluate the thin film stability [8].

In order to determine which of the physical processes has the most significant effect on the nanoparticle formation, the gold film samples of 6 different thicknesses coated on the silicon (110) substrate were prepared and 
irradiated with different pulse energies and the number of laser pulses.

Nanoparticle formation dynamics was investigated in [9] using a single beam treatment of thin gold films. In this paper, we continue our research [9] in addition investigating the nanoparticle formation after irradiation with interfering laser beams. Experimental results using both types of irradiation are compared.

The size of the nanoparticle was found to be weakly dependent on the laser fluence and the number of pulses and was a function of the film thickness for the films thinner than $10 \mathrm{~nm}$. The model, applied to determine the film structure reorganization after irradiation, adequately describes the process of nanoparticle formation. The predicted nanoparticle size and the area density are in good agreement with the experimental results after both types of irradiation (interfered and single beam). Irradiation of the samples with periodically distributed intensity from the interfering laser beams increased regularity in lateral distribution of nanoparticles on the substrate.

\section{Thermal modeling}

Thermal modeling was performed to determine the film melting threshold in order to compare it with a threshold of film morphological changes. The heat conduction model of COMSOL Multiphysics was used to evaluate transient temperature variation during the laser pulse irradiation and in the time span between pulses. A general heat conduction equation used in thermal modeling is shown in Eq. (1).

$$
\rho C_{\mathrm{p}} \frac{\partial T}{\partial t}=\nabla \cdot(\kappa \nabla T)+S
$$

where $\rho=19.3 \mathrm{~g} / \mathrm{cm}^{3}$ is the gold density, $C_{\mathrm{p}}=129 \mathrm{~J} / \mathrm{kg} \mathrm{K}$ is the heat capacity, $\kappa=318 \mathrm{~W} / \mathrm{m} \mathrm{K}$ is the gold thermal conductivity [6]. $S$ is a heat source, which represents the amount of heat gained by absorbing of laser irradiation per unit volume and time $\left(\mathrm{W} / \mathrm{m}^{3}\right)$. The calculation was made for the time covering one laser pulse and the time span between two pulses. Transient temperature variation was evaluated at the point which is in the center of the beam spot on the film surface. Although the film thickness was of the same range as the laser light penetration depth $\delta=13.8 \mathrm{~nm}$, temperature variation in normal direction to the surface can be neglected.

\section{Experimental}

Thin gold films were deposited on polished silicon (110) substrates by using the sputter-coater Q150T ES (Quorum Technologies). Roughness of the film surface was checked with the stylus profiler Dektak 150 (Veeko). The surface roughness of gold thin films was less than $2 \mathrm{~nm}$. Silicon plates with gold films of 6 different thicknesses $(3,4 ; 5,10,15,20,25 \mathrm{~nm})$ were irradiated with the nanosecond laser NL220 (Ekspla Ltd., $\lambda=532 \mathrm{~nm}$, pulse duration FWHM $10 \mathrm{~ns}$ ) operating at the repetition rate of $500 \mathrm{~Hz}$. Angle of incidence was normal to the surface of the sample in all experiments. The laser beam was focused with a quartz lens on the surface of the sample.
The diameter of the Gaussian beam on the surface was $432 \mu \mathrm{m}\left(1 / e^{2}\right)$. The pulse energy was varied from $0.6 \mathrm{~mJ}$ to $2 \mathrm{~mJ}$. Irradiation was performed with the different number of laser pulses in the burst: 1, 10, 100, 1000, 3000, 5000, 10000. Positioning stage Feinmess KDT 180-LM was used to move the sample under the focused laser beam between experiments. Surface morphological changes and the particle formation were observed with the scanning electron microscope (SEM) JSM-6490LV (JEOL). The final size of the nanoparticles was measured with the stylus profiler.

For the experiments with the interference of laser beams, the same nanosecond laser NL 220 (Ekspla) was used. The laser beam was split using a diffractive optical element (DOE). Three beams (angles between beams were 120 degrees in the plane normal to the optical axis) were selected by an aperture. The aperture was necessary to block beams of zero and higher than \pm 1 orders. Split beams were gathered to a spot by a special mirror system for the laser beams interference shown in Fig. 1. The angle of incidence with the optical axis was 24 degrees. The beam was focused with a lens before it was split with DOE. Radius of the area, where interference took place, was 152 $\mu \mathrm{m}$.

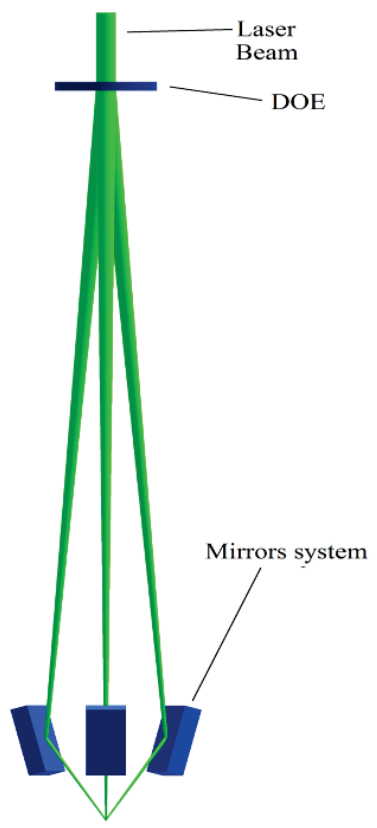

Fig. 1 Experimental scheme for beams interference.

Gold films with the thickness of 4, 5, 6 and $20 \mathrm{~nm}$ on the silicon substrate were irradiated with 3 interfering beams at the focal plane with the different number of pulses ranging from 1 to 1000 and different pulse energy in the range from 8 to $340 \mu \mathrm{J}$.

Nanoparticles on the silicon substrate were analyzed by SEM. The size of nanoparticles and their size distribution were measured and evaluated using UTHSCSA software Image Tools.

\section{Results}

Irradiation of the films with bursts of laser pulses containing the different number of pulses and the laser 
fluence in the center of the beam just above the threshold fluence enabled us to observe the nanoparticle formation dynamics [9].

The morphology of gold films after irradiation with 1 , 10 and 100 pulses with the laser fluence of $200 \mathrm{~mJ} / \mathrm{cm}^{2}$ was investigated by SEM and pictures for the film with the thickness of $5 \mathrm{~nm}$ are shown in Fig. 2.

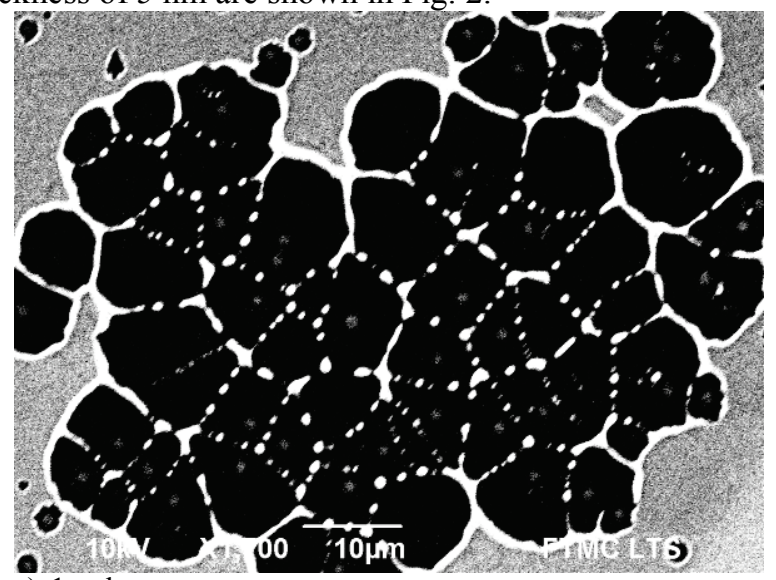

$$
\text { a) } 1 \text { pulse }
$$

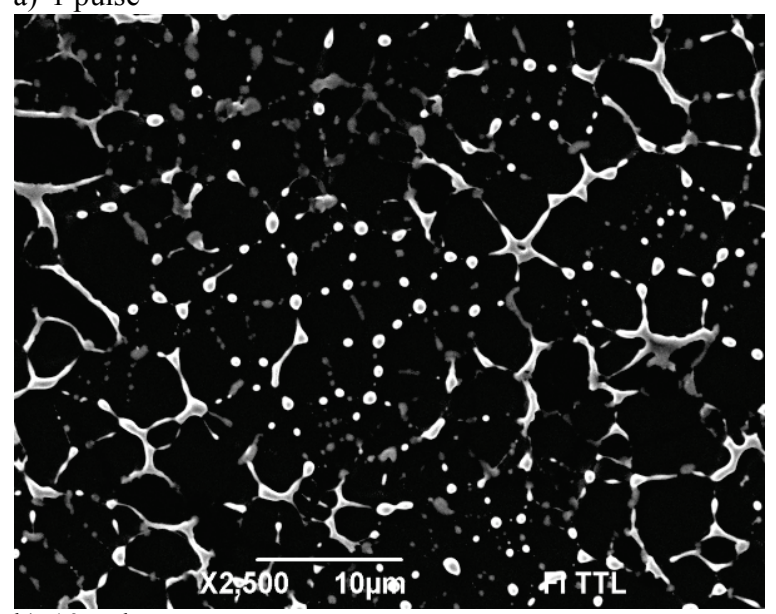

\section{b) 10 pulses}

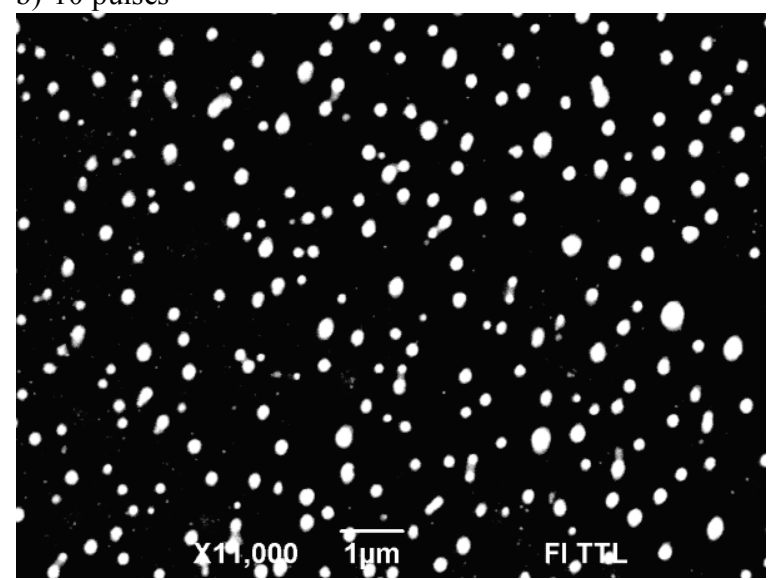

c) 100 pulses

Fig. 2 The morphology of a gold film with the thickness of $5 \mathrm{~nm}$ after laser irradiation with: (a) 1; (b) 10; (c) 100 laser pulses. Laser fluence was $200 \mathrm{~mJ} / \mathrm{cm}^{2}$.

Changes in the film morphology after the first laser pulse are shown in Fig. 2a. The film ruptures spontaneously by forming holes in some spatial order. These holes are growing during the time when the liquid phase exists until metal between holes shrinks to a wire- like network. Image of a film after 10 pulses (Fig. 2b) shows the next step in the particle formation when holes were extended wide enough to touch each other. At the edges of the holes and especially at the contact points of a few holes, metal was collected to nanoparticles. Utilization of a higher number of laser pulses (longer time in a liquid state) led to the nanoparticle formation as is shown in the next SEM image (Fig. 2c). The wires collapsed and nanoparticles with stable nuclei were formed preferentially at crossings of the wires. All these stages are evident depending on the number of laser pulses. The spatial order during the formation process is specific to the dewetting process which has a dominant wavelength for the unstable liquid film perturbation [6]. When nanoparticle formation is induced by nucleation, there is no specific spatial order because the process starts on randomly located defects or contaminations.

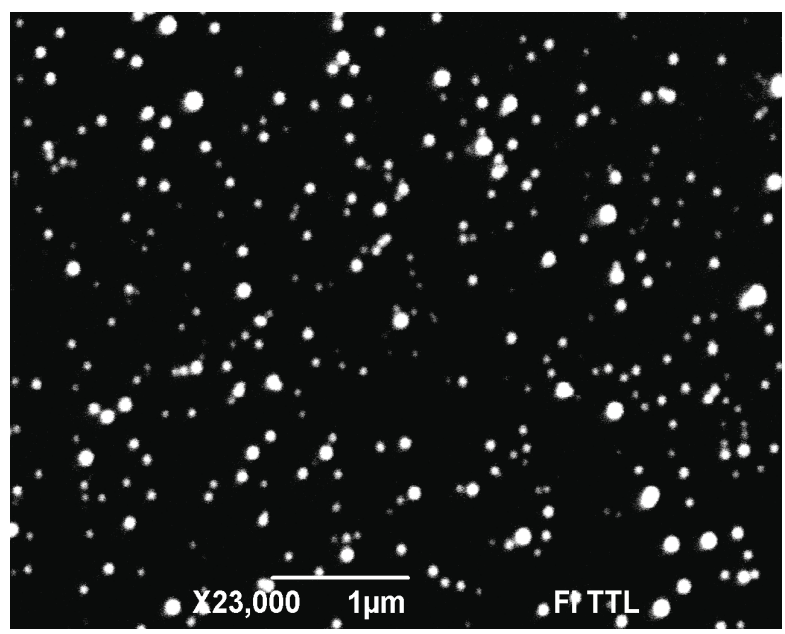

Fig. 3 The morphology of a gold film with the thickness of $3 \mathrm{~nm}$ after irradiation with 1000 laser pulses. Laser fluence was $200 \mathrm{~mJ} / \mathrm{cm}^{2}$.

For the film with a smaller thickness $(3 \mathrm{~nm})$, the final size of nanoparticles was also smaller (Fig. 3). The results indicated that the average diameter of nanoparticles was nearly independent of the number of laser pulses and the pulse energy density [9], as is shown in Fig. 4 and Fig. 5, respectively. Deviations at high laser fluence are probably related to partial evaporation of the smallest nanoparticles increasing the mean size of remaining ones [9].

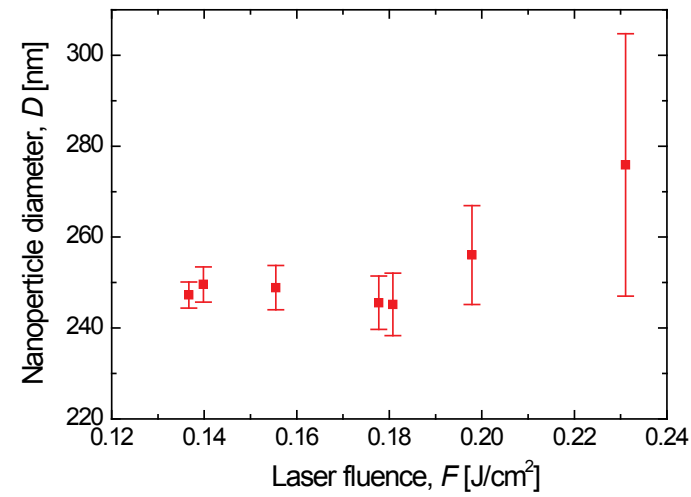

Fig. 4 Mean diameter of nanoparticles depending on the peak laser fluence for the $5 \mathrm{~nm}$-thick $\mathrm{Au}$ film at the end of the formation procedure after irradiation with 1000 laser pulses. 


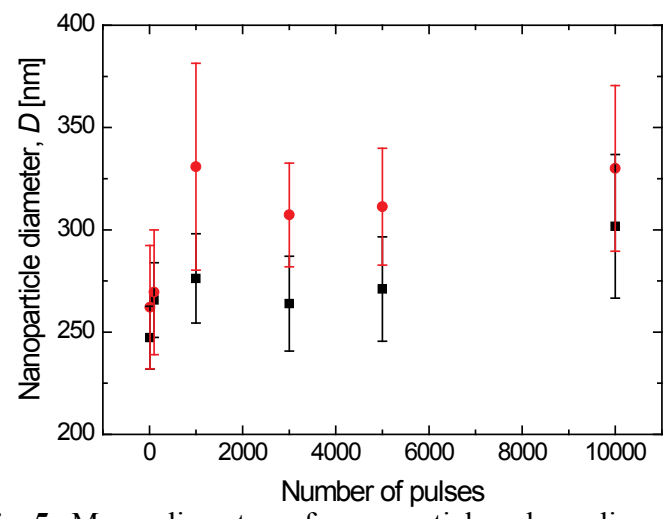

Fig. 5 Mean diameter of nanoparticles depending on the number of laser pulses for the $5 \mathrm{~nm}$ thick Au film. Laser fluence was (•) $190 \mathrm{~mJ} / \mathrm{cm}^{2} ;(\bullet) 220 \mathrm{~mJ} / \mathrm{cm}^{2}$.

This result indicates that the final size of nanoparticles does not depend on the time the film is in a liquid state, which is determined by the number of pulses. In the nucleation process, the stable nuclei grow in time and their growth rate strongly depends on temperature changes. Nanoparticle formation is mostly initiated by the spinoidal dewetting process in the film.

The spinodal dewetting theory for a thin film describes the growth of thickness perturbation of a thin unstable film in time. Such a film is unstable with respect to fluctuations larger than the critical one. The dominating perturbation period can be evaluated from the lubrication approximation of Navier-Stokes equation for a thin film. A dominating perturbation wavelength represents the fastest growing period of the film and is derived from the Navier-Stokes equation. The dominating wavelength can be evaluated from Eq. (2) [10, 11, 12].

$$
\Lambda=\sqrt{\frac{16 \pi^{3} \gamma}{A_{\mathrm{H}}}} h^{2},
$$

here $A_{\mathrm{H}}$ is the Hamaker constant, which contains film-film, film-substrate interaction (interaction between ambient air and substrate; ambient air and film is negligible), $\gamma \approx 1.2 \mathrm{~N} / \mathrm{m}$ is the surface tension of liquid gold at the melting point [13] and $h$ is the film thickness. The total Hamaker constant was approximated using Eq. (3) $[14,15,16]$ :

$$
A_{132} \approx \sqrt{A_{131} A_{232}}
$$

where $A_{131}$ and $A_{232}$ are the individual Hamaker constants for gold and silicon in ambient air, respectively, and they are provided in Table 1.

Table 1. Hamaker constant for gold and silicon in air medium.

\begin{tabular}{cccc}
\hline \hline & Material & $\begin{array}{c}\text { Hamaker } \\
\text { constant, } A_{\mathrm{H}}\end{array}$ & Ref. \\
\hline$A_{131}$ & Gold & $3.110^{-19} \mathrm{~J}$ & {$[17]$} \\
$A_{232}$ & Silicon & $6.510^{-20} \mathrm{~J}$ & {$[18]$} \\
\hline \hline
\end{tabular}

The total Hamaker constant for a gold film on the silicon substrate in ambient air approximated using Eq. (3) and data from Table 1 was $A_{132} \approx 1410^{-20} \mathrm{~J}$.

By analyzing SEM images of samples with the final nanoparticle state it was found that the particle distribution over area on the substrate was proportional to the dominant wavelength. The average distance between two neighboring nanoparticles was a function of the film thickness $R \propto 33.4 h^{2}$. For comparison, the calculated dominant wavelength for the $5 \mathrm{~nm}$ thick film was $650 \mathrm{~nm}$ and the average distance between two neighboring particles was as long as $632 \mathrm{~nm}$.

The nanoparticle diameter can be found theoretically. Assuming that there are no losses of the metal due to evaporation from the substrate during the laser irradiation, a volume of a single particle includes the volume of the film from a square area with dimensions of the dominant wavelength (Fig. 6). The height of nanoparticles was measured with the profiler. Comparing the mean nanoparticle diameter determined by SEM images analysis and the average nanoparticle height, we came to the conclusion that nanoparticles were of nearly semispherical shape, which is reasonable due to surface tensions in a droplet. Using Eq. (4), the nanoparticle diameter can be estimated.

$$
D=\left[\frac{192 \pi^{2} \gamma}{A_{\mathrm{H}}}\right]^{\frac{1}{3}} h^{\frac{5}{3}} .
$$

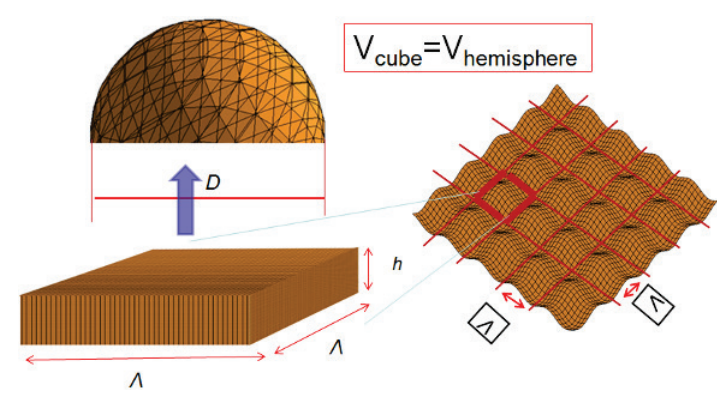

Fig. 6 Scheme of nanoparticle formation after the thin liquid film became unstable.

The experimental and theoretical diameters of nanoparticles as a function of the film thickness are given in Fig. 7.

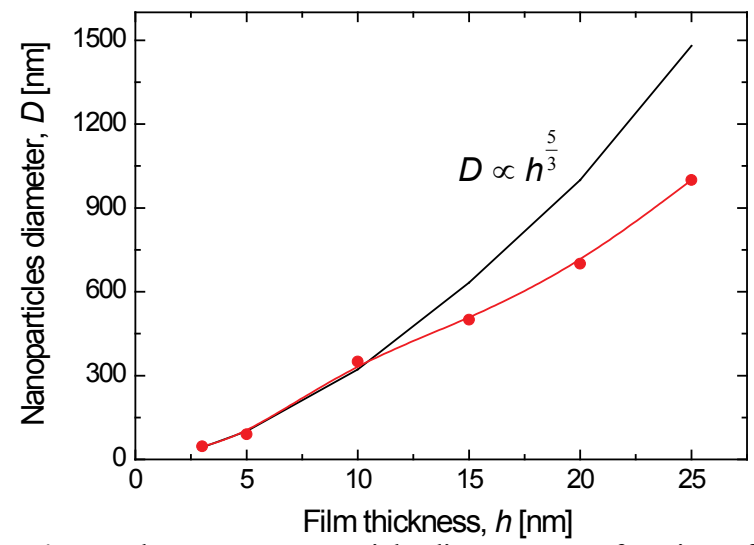

Fig. 7 The mean nanoparticle diameter as a function of the film thickness after irradiation with 1000 pulses, mean laser fluence $192.2 \mathrm{~mJ} / \mathrm{cm}^{2}:(\bullet)$ experimental results; solid line was calculated using Eq. (4).

Curves shown in Fig. 7 coincide well for the films with the thickness up to $15 \mathrm{~nm}$. In the range of the film 
thickness larger than $15 \mathrm{~nm}$, the dewetting theory does not show a good agreement with the experimental data to explain the particle formation. In gold films with a large thickness, the thermal gradient appears to be normal to the surface direction. Therefore, the range of intermolecular dispersive forces which cause the perturbation in the film becomes shorter than the film thickness.

The area density of nanoparticles is an important parameter of the technological process, which determines the number of nanoparticles per unit substrate area. The area density of particles was calculated using Eq. (5).

$$
N=\frac{1}{\Lambda^{2}}=\frac{A_{\mathrm{H}}}{16 \pi^{3} \gamma} h^{-4} .
$$

The averaged area density was experimentally evaluated by analyzing SEM images with the software "Image tool". Experimental and calculated area density of the gold nanoparticles on a logarithmic scale for various film thicknesses is given in Fig. 8 .

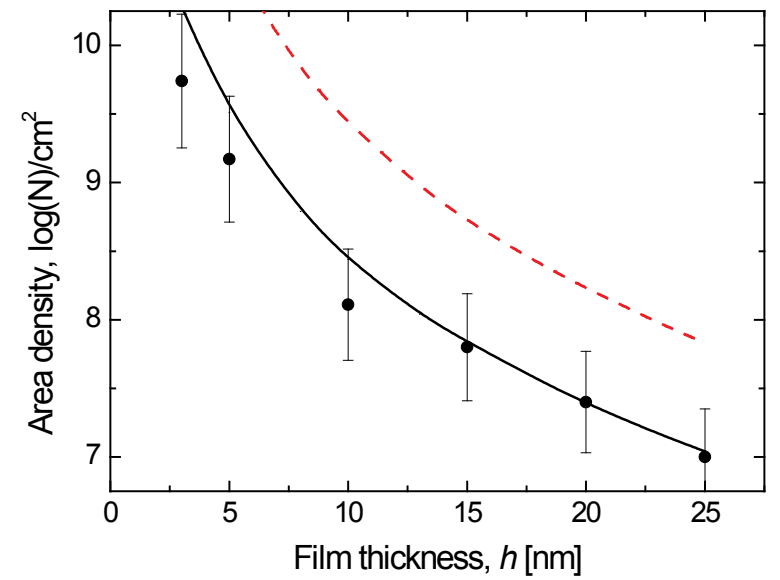

Fig. 8 The area density of nanoparticles on a logarithmic scale: $(\bullet)$ experimental points; red dashed line calculated by using Eq.(5); black solid line calculated by using Eq. (8).

The values of the theoretical area density calculated by using Eq. (5) differ from the experimental data (Fig. 8). Under our experimental conditions, the metal partially vaporizes during the laser heating process. The vaporization flux depends on temperature and can be expressed as [19]:

$$
\Gamma_{\mathrm{e}}=\sqrt{\frac{M}{2 \pi R T}} P_{\mathrm{e}},
$$

here $M$ is the gold molar mass, $R$ is the universal gas constant, $T$ is temperature and $P_{\mathrm{e}}$ is the equilibrium vapor pressure of gold at a certain temperature. In the nonequilibrium case, any temperature changes cause the vapor pressure changes. The variable $P_{\mathrm{e}}(T)$ is expressed as temperature dependent. In order to determine the number of particles per second lost due to evaporation of gold, the evaporated volume of the metal should be estimated and divided by the volume of all nanoparticles. The correction coefficient can be expressed as the amount of the lost nanoparticles through evaporation process:

$$
K_{l}=\int_{0}^{t} \sqrt{\frac{M}{2 \pi R T}} \frac{P(T)}{\pi \rho D(h)^{2}} \mathrm{~d} t .
$$

The area density of nanoparticles is as follows:

$$
N(h, t)=\frac{A_{\mathrm{H}}}{16 \pi^{3} \gamma} h^{-4}-K_{l} .
$$

Eq. (8) was solved two-dimensionally by using the finite element method in Maple 14 software. Values for $P_{\mathrm{e}}(T)$ were taken from [20]. The area density of nanoparticles calculated using Eq. (8) is given as a solid black line in Fig. 8. The theoretical model which includes the dewetting process and evaporation of a thin film coincides well with the experimental results.

\section{Gold films irradiation with interfering laser beams}

Nanoparticle formation from thin gold film was also investigated using the 3-beams laser interference. Laser patterning with the several beam interference is capable of producing sub-wavelength features not limited by the spot size and is an effective method of producing $2 \mathrm{D}$ or $3 \mathrm{D}$ periodic structures [21]. The periodical structure can be controlled by changing the incidence angle of the beams, wavelength of radiation, phase difference between the beams, polarization and intensity.

The first result showed that the diameter distribution of particles after irradiation by the interfering field was much narrower than using the single beam treatment. The comparison of the nanoparticle distributions in size is shown in Fig. 9.

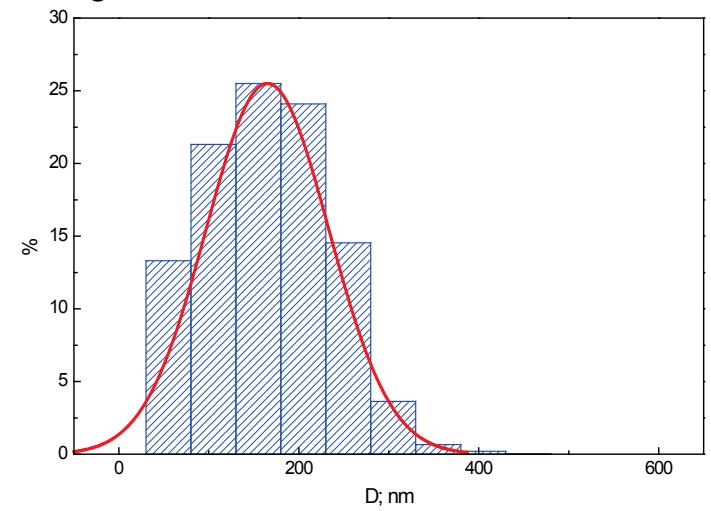

a)

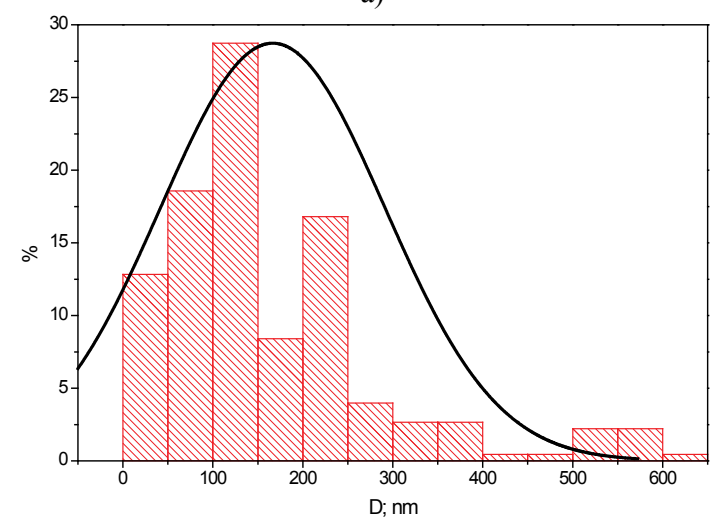

b)

Fig. 9 Nanoparticle diameter distribution after irradiation of the $5 \mathrm{~nm}$ gold layer. a) Irradiation with interfering laser beams, average fluence $55 \mathrm{~mJ} / \mathrm{cm}^{2}$ and 100 pulses, b) single beam treatment, average fluence $191 \mathrm{~mJ} / \mathrm{cm}^{2}$ and 100 pulses. Fitting curves (normal function) are superimposed on the histograms. 
Another significant difference of the nanoparticles generated with the interfering laser beams is that nanoparticles are distributed in a higher order than after the single beam treatment (see Fig. 10 and Fig. 12). The reason for this periodicity is related with temperature variation of the film surface. The intensity distribution of 3 interfering laser beams is periodical. This distribution was modeled with MAPLE and is presented in Fig 11. The period of the interference intensity at the focal plane was $0.6 \mu \mathrm{m}$ using parameters of out experimental setup. The temperature distribution as well as the intensity on a film are periodical. The Marangoni force starts to act the film after the temperature gradient appears in close area. This force drives a molten material from the areas with higher to lower temperature [22].

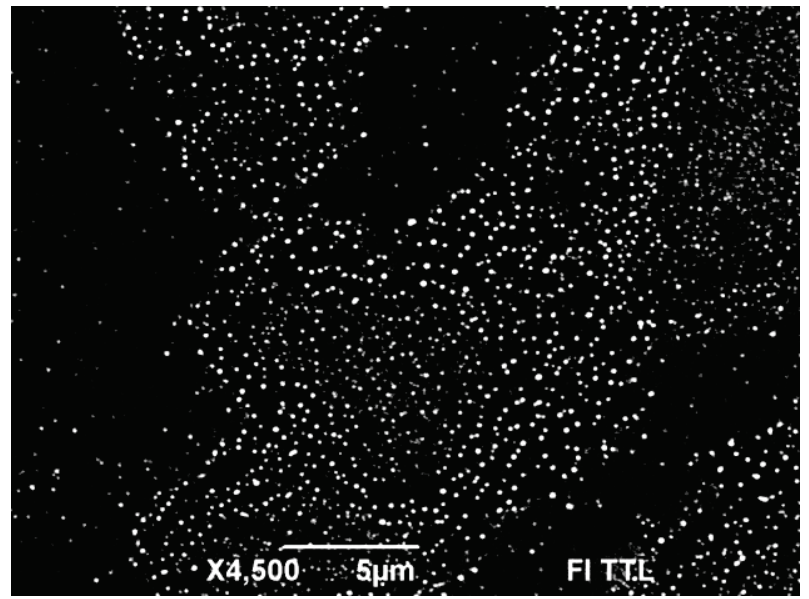

a) Single beam irradiation;

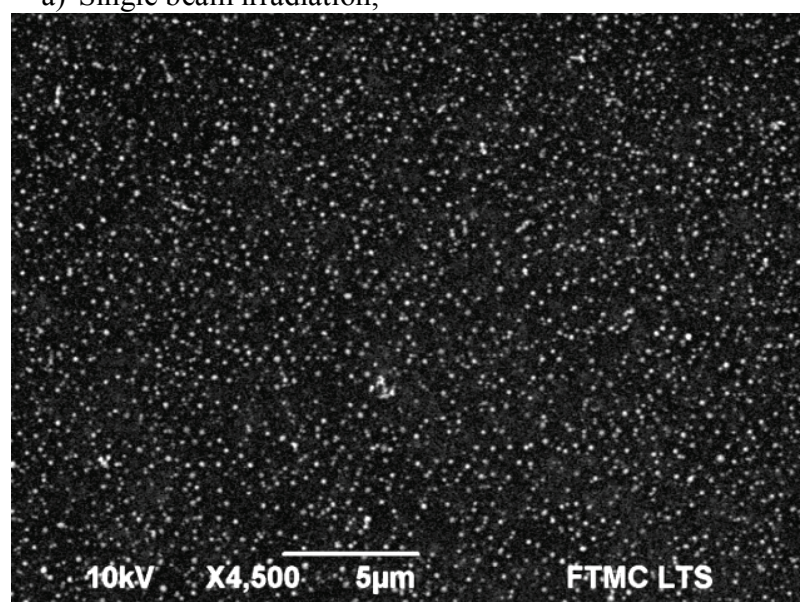

b) Irradiation by the interfering laser beams.

Fig. 10 Nanoparticles distribution after irradiation with a single beam at the mean laser fluence was $55.5 \mathrm{~mJ} / \mathrm{cm}^{2}$ (a) and the 3-beam interference irradiation, mean laser fluence was 192.1 $\mathrm{mJ} / \mathrm{cm}^{2}$ (b). Film thickness was $5 \mathrm{~nm}$. (Note that peak laser fluence in case of 3 interfering beams is 3 times higher than average).

After irradiation with the interfering laser beams, the particles were spatially ordered and this could be influenced by the Marangoni force initiated molten metal motion. As it is shown in Fig. 10b and Fig. 12b after irradiation of a film, particles formed a periodic structure and the period coincided with the period of the interference intensity and was approximately equal to $0.6 \mu \mathrm{m}$.

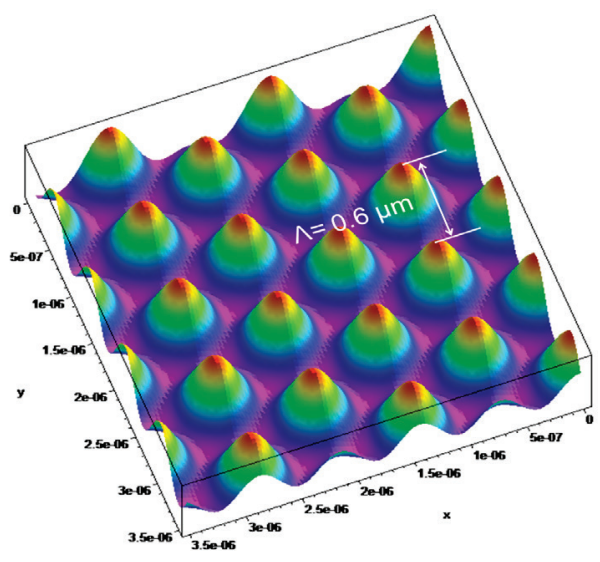

Fig. 11 Intensity distribution of the 3-beam interference, modeled with MAPLE.

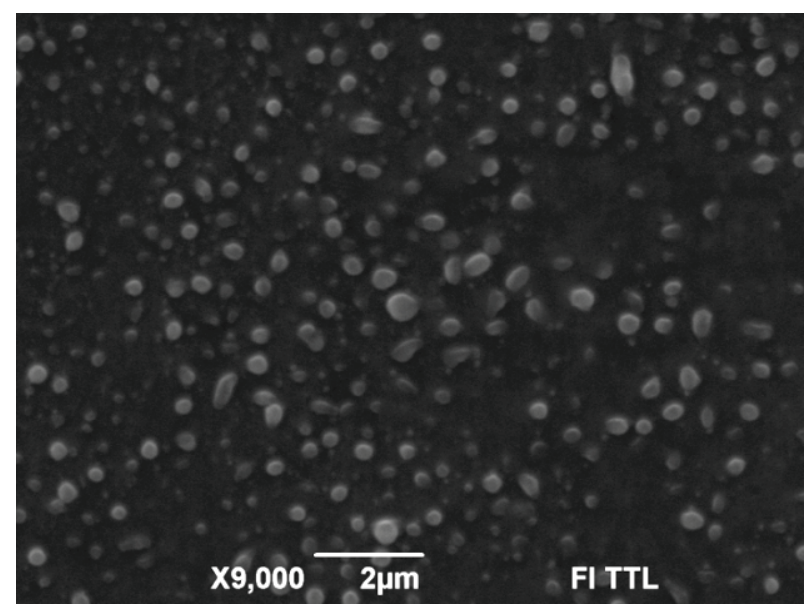

a) Single beam irradiation;

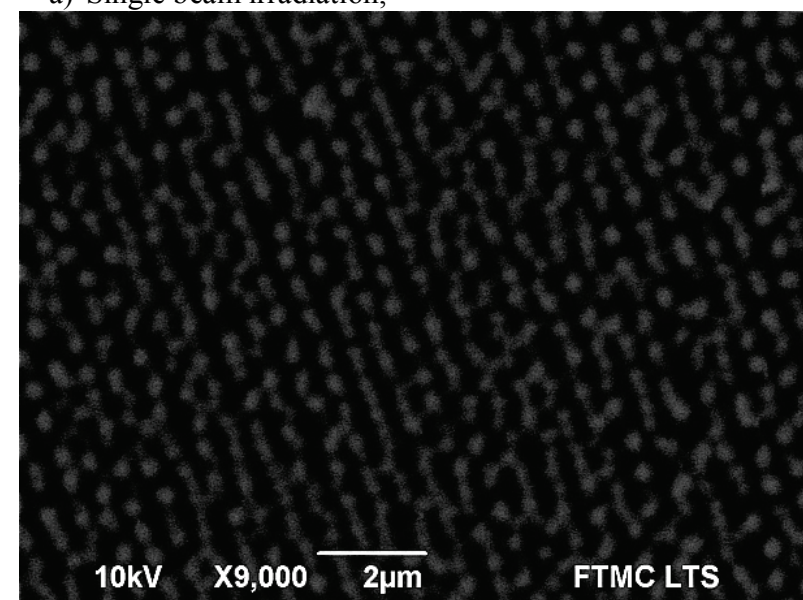

b) Irradiation with the interfering laser beams

Fig. 12 Nanoparticles distribution after irradiation with a single beam at the mean laser fluence was $55.5 \mathrm{~mJ} / \mathrm{cm}^{2}$ (a) and the 3-beam interference irradiation, mean laser fluence was 192.1 $\mathrm{mJ} / \mathrm{cm}^{2}$ (b). Film thickness was $20 \mathrm{~nm}$.

The next result indicated that the average nanoparticle diameter mostly depended on the film thickness after irradiation by interfering laser beams as well as after a single-beam irradiation. For comparison, the average diameter of particles was $166.5 \mathrm{~nm}$ after the single beam irradiation and $165.1 \mathrm{~nm}$ in case of irradiation with the interfering laser beams for the film with thickness of $4 \mathrm{~nm}$. Dependence of the average particle diameter on the film 
thickness for both irradiation schemas and results of simulations according to Eq. (4) are presented in Fig. 13.

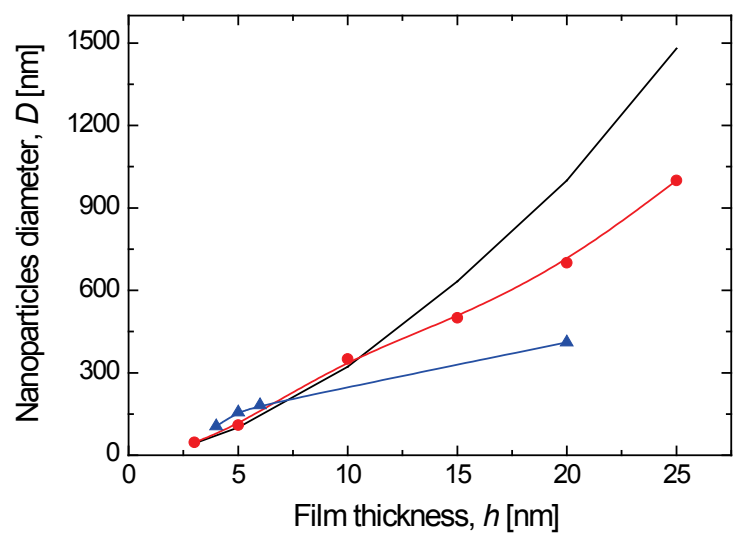

Fig. 13 The mean nanoparticle diameter as a function of the film thickness. The pulse number 100 , average fluence $192.2 \mathrm{~mJ} / \mathrm{cm}^{2}$ for a single beam $(\bullet)$ and $55.5 \mathrm{~mJ} / \mathrm{cm}^{2}$ for the irradiation with 3 interfering laser beams $(\boldsymbol{\Delta})$. Simulation results according Eq.(4) are given as the black curve. The periodic interference field distribution leads to formation of smaller nanoparticles in the films with thickness larger than $10 \mathrm{~nm}$.

Experimental data after irradiation with the interfering and single beam demonstrate very similar results in size of nanoparticles for the films several nanometers thick. Differences for thicker films arrive from input of Marangoni convection which is stronger in case of the laser beam interference as the size of a single maximum in the periodic field distributions is significantly smaller the laser beam spot used in the single-beam experiments.

\section{Conclusions}

Formation of nanoparticles in thin gold films on the silicon substrate after laser irradiation is caused by the dewetting phenomenon. The average diameter of the nanoparticles varies with the film thickness $h$ as a function $D \propto h^{5 / 3}$ of dominant wavelength of the dewetting process down to thickness of $10 \mathrm{~nm}$, and can be evaluated from the film and irradiation parameters. For the films thicker than $10 \mathrm{~nm}$, experimentally measured nanoparticles diameters do not coincide with the calculated values using the dewetting theory. The reason is that for a gold film thicker than $10 \mathrm{~nm}$, the film thickness exceeds the range of intermolecular dispersive forces. Therefore, the thermal gradient appears in the direction normal to the film surface. By estimating the area density of nanoparticles, the correction coefficient due to evaporation losses of the metal should be taken in account. The calculated area density by using the correction coefficient coincides well with the experimental values.

Thin layer structuring with interfering laser beams provides the area distribution of nanoparticles in a higher spatial order. Therefore, the width of the particle size distribution becomes narrower compared to the single beam irradiation method.

\section{Acknowledgment}

The work was supported by the Research Council of Lithuania under grant No. ATE-11/2012.

\section{References}

[1] S. Y. Lee, M. Yamada and M. Miyaki: Carbon, 43 (2005) 2654.

[2] L. C. Campos, V. R. Manfrinato, J. D. SanchezYamagishi, J. Kong and P. Jarillo-Herrero: Nano Lett., 9 (2009) 2600.

[3] P. Ghosh, G. Han, M. De, C. K. Kim, V. M. Rotello: Adv. Drug Delivery Rev., 60 (2008) 1307.

[4] X. Cao, Y. Ye and S. Liu: Anal. Biochem., 417 (2011) 1.

[5] D. Wang, R. Ji, P. Schaaf: Beilstein J. Nanotechnol., 2 (2011) 318.

[6] C. Favazza, R. Kalyanaraman and R. Sureshkumar: Nanotechnol., 17 (2006) 4229.

[7] S. J. Henley, J. D. Carey and S. R. P. Silva: Appl. Surf. Sci., 253, (2007) 8080.

[8] F. Flandoli: "Random Perturbation of PDEs and Fluid Dynamic Models" 1st ed. By Applicata (Springer, Berlin, 2010).

[9] K. Ratautas, M. Gedvilas, G. Račiukaitis, A. Grigonis: J. Appl. Phys., 112 (2012) xx.

[10]A. Vrij: Discuss. Faraday Soc., 42 (1966) 23.

[11]A. Sharma and E. Ruckenstein: Langmuir, 2 (1986) 480.

[12]C. Favazza, H. Krishna, R. Sureshkumar and R. Kalyanaraman: Proc. SPIE, 6648 (2007) 664809.

[13]N. Eustathopoulos, B. Drevet and E. Ricci: J. Crystal Growth, 191 (1998) 268.

[14] J. N. Israelachvili: "Intermolecular and Surface Forces" 2nd ed., (Academic, London, 1992).

[15]H.-J. Butt and M. Kappl: "Surface and Interfacial Forces" 1st ed., (Wiley-VCH, Weinheim, 2010).

[16] K. M. Hurst, C. B. Roberts and W. R. shurst: Nanotechnol., 20 (2009) 185303.

[17]D. Bargeman and F. V. V. Vader: J. Electroanal. Chem., 37 (1972) 45.

[18]D. Bachmann, S. Kuhne and C. Hierold: Sensors Actuators A, 132 (2006) 407.

[19] S. Fujikawa, T. Yano and M. Watanabe: "Handbook of Liquid Vapor Interfaces, Bubbles and Droplets" 1st ed., (Springer, New York, 2008).

[20]D. L. Hildebrand and W. F. Hall: Phys. Chem., 66 (1962) 754.

[21]B. Voisiat, M. Gedvilas, S. Indrišiūnas, G. Račiukaitis: Phys. Procedia, 12 (2011) 116.

[22]M. Gedvilas, B. Voisiat, G. Račiukaitis, K. Regelskis: Appl. Surf. Sci., 255 (2009) 9826.

(Received: June 29, 2012, Accepted; October 26, 2012) 